\title{
AN INTEGRAL INVOLVING A MODIFIED BESSEL FUNCTION OF THE SECOND KIND AND AN $E$-FUNCTION
}

\author{
by FOUAD M. RAGAB
}

(Received 30th April, 1951)

§ 1. Introductory. The formula to be established is

$$
2^{m+1} m \pi^{m-1} \int_{0}^{\infty} K_{m n}(2 m \lambda) \lambda^{m k-1} E\left(p ; \alpha_{r}: q ; \rho_{s}: \frac{x}{\lambda^{2 m}}\right) d \lambda=E\left(p+2 m ; \alpha_{r}: q ; \rho_{s}: x\right), \ldots
$$

where $m$ is a positive integer,

$$
\left.\begin{array}{l}
\alpha_{p+2 \nu+1}=\frac{1}{2} k+\frac{1}{2} n+\frac{\nu}{m} \\
\alpha_{p+2 \nu+2}=\frac{1}{2} k-\frac{1}{2} n+\frac{\nu}{m}
\end{array}\right\} \nu=0,1,2, \ldots, m-1,
$$

and the constants are such that the integral converges.

This formula was proved by induction for the case $m=2^{l}, l=1,2,3, \ldots$, (1).

The following formulae are required in the proof :

$$
4 \int_{0}^{\infty} K_{n}(2 \lambda) \lambda^{k-1} E\left(p ; \alpha_{r}: q ; \rho_{s}: \frac{x}{\lambda^{2}}\right) d \lambda=E\left(p+2 ; \alpha_{r}: q ; \rho_{s}: x\right),
$$

where $\alpha_{p+1}=\frac{1}{2} k+\frac{1}{2} n, \alpha_{p+2}=\frac{1}{2} k-\frac{1}{2} n, R(k \pm n)>0(2)$;

$$
\prod_{\nu=1}^{m-1} \int_{0}^{\infty} K_{n}(2 t) t_{\nu}^{2 \nu / m-1} d t_{\nu} K_{n}\left(\frac{2 b}{t_{1} t_{2} \ldots t_{m-1}}\right)=\left(\frac{\pi}{2}\right)^{m-1} K_{m n}\left(2 m b^{1 / m}\right)
$$

where $b>0,(3)$.

§2. Proof of the Integral. Apply formula (2) repeatedly to itself, taking for $k$ the values $k+2 v / m, v=1,2, \ldots, m-1:$ then

$$
\begin{gathered}
4^{m} \int_{0}^{\infty} K_{n}(2 \lambda) \lambda^{1 \sigma-1} d \lambda \prod_{\nu=1}^{m-1} \int_{0}^{\infty} K_{n}\left(2 t_{\nu}\right) t_{\nu}^{k+2 \nu / m-1} d t_{\nu} E\left(p ; \alpha_{r}: q ; \rho_{s}: \frac{x}{\lambda^{2} t_{1}{ }^{2} \ldots t^{2}{ }_{m-1}}\right) \\
=E\left(p+2 m ; \alpha_{r}: q ; \rho_{s}: x\right),
\end{gathered}
$$

where the $\alpha$ 's after $\alpha_{p}$ are given by $\left(1^{\prime}\right)$.

Here, on the left, put the first integral last and then put $\lambda=\mu /\left(t_{1} t_{2} \ldots t_{m-1}\right)$; the expression becomes

$$
4_{\nu=1}^{m-1} \int_{0}^{\infty} K_{n}\left(2 t_{\nu}\right) t_{\nu}^{2 \nu / m-1} d t_{\nu} \int_{0}^{\infty} K_{n}\left(\frac{2 \mu}{t_{1} t_{2} \ldots t_{m-1}}\right) \mu^{k-1} E\left(p ; \alpha_{r}: q ; \rho_{s}: \frac{x}{\mu^{2}}\right) d \mu .
$$

Now change the order of integration so that the last integral becomes the first integral, and apply (3) ; this gives

$$
2^{m+1} \pi^{m-1} \int_{0}^{\infty} K_{m n}\left(2 m \mu^{1 / m}\right) \mu^{k-1} E\left(p ; \alpha_{r}: q ; \rho_{s}: \frac{x}{\mu^{2}}\right) d \mu
$$

On putting $\mu=\lambda^{m}$, formula (1) is obtained.

Corollary. On replacing $\lambda$ by $\lambda / i$ and $x$ by $x e^{-i m \pi}$ in (1) and making use of the formula

$$
K_{n}(t)=i^{n} G_{n}(i t)
$$


it is found that

$$
\begin{aligned}
2^{m+1} m \pi^{m-1} \int_{0}^{\infty} G_{m n}(2 m \lambda) \lambda^{m k-1} E\left(p ; \alpha_{r}: q ; \rho_{s}: \frac{x}{\lambda^{2 m}}\right) d \lambda & \\
& =i^{m(k-n)} E\left(p+2 m ; \alpha_{r}: q ; \rho_{s}: x e^{-i m \pi}\right),
\end{aligned}
$$

where $k \pm n>0, m\left(2 \alpha_{r}-k\right)>-\frac{3}{2}, r=1,2, \ldots, p$.

Similarly, on replacing $\lambda$ by $\lambda i$ and $x$ by $x e^{i m \pi}$, it is found that

$$
\begin{aligned}
2^{m+1} m \pi^{m-1} \int_{0}^{\infty} G_{m n}\left(2 m \lambda e^{i \pi}\right) \lambda^{m k-1} E\left(p ; \alpha_{r}: q ; \rho_{s}: \frac{x}{\lambda^{2 m}}\right) d \lambda \\
\cdot=i^{-m(k+n)} E\left(p+2 m ; \alpha_{r}: q ; \rho_{s}: x e^{i m \pi}\right),
\end{aligned}
$$

where $k \pm n>0, m\left(2 \alpha_{r}-k\right)>-\frac{3}{2}, r=1,2, \ldots, p$.

From (5) and (6), and making use of the formula

it follows that

$$
\pi i J_{n}(x)=G_{n}(x)-i^{2 n} G_{n}\left(x e^{i \pi}\right),
$$

$$
\begin{aligned}
2^{m+1} m \pi^{m} i \int_{0}^{\infty} J_{m n}(2 m \lambda) \lambda^{m k-1} E\left(p ; \alpha_{r}: q ; \rho_{s}: \frac{x}{\lambda^{2 m}}\right) d \lambda \\
=i^{m(k-n)} E\left(p+2 m ; \alpha_{r}: q ; \rho_{s}: x e^{-i m \pi}\right) \\
\quad-i^{-m(k-n)} E\left(p+2 m ; \alpha_{r}: q ; \rho_{s}: x e^{i m \pi}\right),
\end{aligned}
$$

where the $\alpha$ 's are given by $\left(1^{\prime}\right)$ and $k+n>0, m\left(2 \alpha_{r}-k\right)>-\frac{3}{2}, r=1,2, \ldots, p$.

\section{REFERENCES}

(1) Ragab, F. M., Proc. Glasg. Math. Ass., 1, 74 (1952).

(2) MacRobert, T. M., Phil. Mag. (vii), 31, 258 (1941).

(3) Ragab, F. M., Proc. Glasg. Math. Ass., 1, 115 (1953).

\section{University of Glasgow}

